International Journal of Bifurcation and Chaos, Vol. 10, No. 9 (2000) 2221-2231

(C) World Scientific Publishing Company

\title{
ORDERING CHAOS IN CHUA'S CIRCUIT: A SAMPLED-DATA FEEDBACK AND DIGITAL REDESIGN APPROACH*
}

\author{
SHU-MEI GUO, LEANG S. SHIEH, \\ GUANRONG CHEN and MARITZA ORTEGA \\ Department of Electrical and Computer Engineering, \\ University of Houston, Houston, TX 77204-4793, USA
}

Received October 4, 1999; Revised October 26, 1999

\begin{abstract}
In this paper, we develop and apply some digital design and redesign techniques for ordering the chaotic Chua's circuit. The idea of using sampled-data feedback for controlling the circuit was previously suggested [Yang \& Chua, 1998], which relies on small sampling periods. We show how this sampled-data feedback control method can be significantly improved, so that large sampling times are allowed, for the same purpose of ordering the nonlinear circuit, from anywhere within the chaotic attractor towards a predesired periodic cycle of the circuit.
\end{abstract}

\section{Introduction}

Within the areas of dynamics and control for nonlinear systems, the research on controlling chaos has seen a dramatic increase in the last decade (see e.g. [Chen \& Dong, 1998; Chen, 1999; Chen \& Ogorzalek, 2000]).

There have been many successful methods for controlling or ordering chaos, many of which make use of some essential properties of chaotic systems such as their sensitivity to initial conditions, the feature of possessing a dense set of unstable periodic orbits of different periods, and the dependence on various bifurcations. In particular, generating chaos via control techniques, when chaos is useful and beneficial, utilizes the very features of chaos and the essence of feedback control principles [Chen \& Dong, 1998].

It is now known that controlling chaos in continuous-time systems can be implemented by analog circuits. However, in order to take advantage of the modern high-speed computers and microelectronics, it is more preferable to use digital controllers instead of analog circuits, particularly in aerospace systems and industries [Dedieu \& Ogorzalek, 1994; Yang \& Chua, 1998].

The process of converting an existing continuous-time controller to an equivalent discretetime controller is called digital redesign [Shieh et al., 1989, 1991, 1992a, 1992b; Tsai et al., 1989, 1991]. Digital redesign is generally technical, since there are some critical issues such as the sensitive instability problem existing in the redesign when the desired short sampling periods are used in digitalization (i.e. if the sampling time is too short then it can be very CPU time-consuming and may cause instability in its digital version of the control system). Moreover, fast-rate sampling devices can be very expensive or even physically impossible. For these reasons, digital redesign techniques have never been applied to nonlinear systems, with perhaps the only exception [Xu et al., 1996], particularly the numerically extremely sensitive chaotic systems like Chua's circuit.

Digital redesign was first studied in detail by Kuo [1980]. He proposed a discrete-state

* ${ }^{*}$ Supported by the US Army Research Office under Grant DAAG55-98-1-0198. 
matching method and applied it to a simplified one-axis sky-lab satellite system. Recently, Shieh and his colleagues [Shieh et al., 1989, 1991, 1992a, 1992b; Tsai et al., 1989, 1991] have thoroughly investigated this type of digital redesign and various types of (sub)optimal digital redesign methods, so that the digital redesign technology is greatly advanced.

In this paper, we develop and apply the successful digital redesign techniques to the ordering of the chaotic Chua's circuit. We will show how the sampled-data feedback control method using a small sampling time [Yang \& Chua, 1989] can be significantly improved to be an even better digital controller, with large sampling times. Our approach is again to first develop a successful analog controller, for controlling the chaotic trajectories of Chua's circuit, and then to redesign it via advanced digital control technique, so as to control the circuit dynamics from anywhere within its chaotic attractor to a predesired periodic trajectory.

The material presented in this paper is organized as follows. First, a very brief introduction to Chua's circuit is given, under the format to be used in the digital redesign framework of this paper. Then, an existing continuous-time feedback control approach for controlling the chaotic Chua's circuit is described. To that end, an appropriate digitalization of the continuous-time controller is suggested. The digitally controlled Chua's circuit is then studied. The behaviors of the digitally controlled Chua's circuit with different sampling periods are compared by computer simulations. Finally, a good digital redesign method for improving the critical control performance of a long samplingperiod digital controller for the circuit is introduced and simulated.

\section{The Chaotic Chua's Circuit}

Chua's circuit is a simple electronic circuit that exhibits a wide variety of nonlinear dynamical phenomena such as bifurcations and chaos. This circuit, as shown in Fig. 1(a), can be described by the following set of dynamical equations:

$$
\begin{aligned}
& \frac{d v_{1}}{d t}=\frac{1}{C_{1}}\left[G\left(v_{2}-v_{1}\right)-f\left(v_{1}\right)\right], \\
& \frac{d v_{2}}{d t}=\frac{1}{C_{2}}\left[G\left(v_{1}-v_{2}\right)+i_{L}\right], \\
& \frac{d i_{L}}{d t}=-\frac{1}{L} v_{2},
\end{aligned}
$$

where $G=1 / R, R$ is a resistor, $i_{R}$ is the current through the inductor $L, v_{1}$ and $v_{2}$ are the voltages across the capacitors $C_{1}$ and $C_{2}$, respectively, and

$$
\begin{aligned}
f\left(v_{1}\right)= & G_{b} v_{1} \\
& +\frac{1}{2}\left(G_{a}-G_{b}\right)\left(\left|v_{1}+E\right|-\left|v_{1}-E\right|\right)
\end{aligned}
$$

with $E$ being the breakpoint voltage of the diode, and $G_{b}<0$ and $G_{a}<0$ being some appropriate

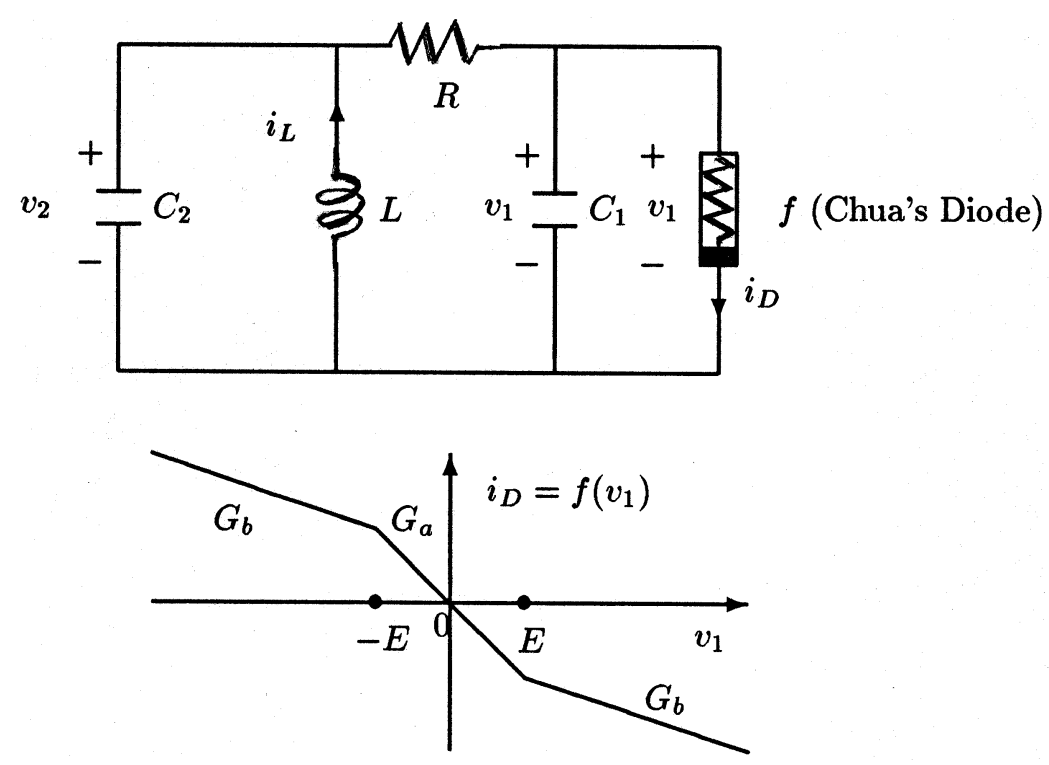

Fig. 1. Chua's circuit: (a) the circuitry; (b) the piecewise-linear resistance. 
constants, representing slopes of the piecewiselinear resistance. by

The dimensionless version of the circuit is given

$$
\left\{\begin{array}{l}
\dot{x_{1}}=\alpha\left(-x_{1}+x_{2}-f\left(x_{1}\right)\right) \\
\dot{x_{2}}=x_{1}-x_{2}+x_{3} \\
\dot{x_{3}}=-\beta x_{2}
\end{array}\right.
$$

where $x_{1}=v_{1} / E, x_{2}=v_{2} / E, x_{3}=i_{R} /(E G)$, $\alpha=C_{2} / C_{1}$, and $\beta=1 /(L G)=C_{2} R^{2} / L$ by letting $G=C_{2}$, and

$$
\begin{aligned}
f\left(x_{1}\right) & =m_{0} x_{1}+0.5\left(m_{1}-m_{0}\right)\left(\left|x_{1}+1\right|-\left|x_{1}-1\right|\right) \\
& = \begin{cases}m_{0} x_{1}+m_{1}-m_{0} & x_{1} \geq 1.0 \\
m_{1} x_{1} & \left|x_{1}\right| \leq 1.0 \\
m_{0} x_{1}-m_{1}+m_{0} & x_{1} \leq-1.0\end{cases}
\end{aligned}
$$

in which $m_{0}=R G_{b}$ and $m_{1}=R G_{a}$. This piecewise-linear function is shown in Fig. 1(b), with $E=1$ therein.

Now, putting the circuit equation into the state-space format, we have the following systems, which are continuously connected together:

$$
\dot{x}(t)=A_{i} x(t)+B_{i} u_{i}(t), \quad i=1,2,3,
$$

with the linear segments contained in $\left(A_{i}, B_{i}\right)$ for $i=1,2,3$ in (6)-(8), respectively:

$$
\begin{aligned}
& \dot{x}(t)=\left[\begin{array}{ccc}
-\alpha\left(1+m_{0}\right) & \alpha & 0 \\
1 & -1 & 1 \\
0 & -\beta & 0
\end{array}\right] x(t) \\
& +\left[\begin{array}{ccc}
-\alpha\left(m_{1}-m_{0}\right) & 0 & 0 \\
0 & 1 & 0 \\
0 & 0 & 1
\end{array}\right] u_{1}(t), \\
& x_{1}(t)>1 \text {, } \\
& \dot{x}(t)=\left[\begin{array}{ccc}
-\alpha\left(1+m_{1}\right) & \alpha & 0 \\
1 & -1 & 1 \\
0 & -\beta & 0
\end{array}\right] x(t) \\
& +\left[\begin{array}{lll}
1 & 0 & 0 \\
0 & 1 & 0 \\
0 & 0 & 1
\end{array}\right] u_{2}(t), \quad\left|x_{1}(t)\right| \leq 1,
\end{aligned}
$$

$$
\begin{gathered}
\dot{x}(t)=\left[\begin{array}{ccc}
-\alpha\left(1+m_{0}\right) & \alpha & 0 \\
1 & -1 & 1 \\
0 & -\beta & 0
\end{array}\right] x(t) \\
+\left[\begin{array}{ccc}
\alpha\left(m_{1}-m_{0}\right) & 0 & 0 \\
0 & 1 & 0 \\
0 & 0 & 1
\end{array}\right] u_{3}(t), \\
x_{1}(t)<-1 .
\end{gathered}
$$

With $\alpha=9, \beta=14 \frac{2}{7}, m_{0}=-5 / 7, m_{1}=$ $-8 / 7, u_{1}(t)=u_{3}(t)=(1,0,0)^{\top}$ and $u_{2}(t)=$ $(0,0,0)^{\top}$, the chaotic trajectory of the circuit, $x_{c i}(t)$, is shown in Figs. 2-4, where the initial point $(-0.1,-0.1,-0.1)^{\top}$ was used in the simulation. In these figures, the approximate limit cycle $\bar{x}_{i}(t)$ is calculated by (14) and the continuous controller used is given by (15), as will be seen below. Note that $B_{2} u_{2}(t)=\bar{B}_{2} \bar{u}_{2}(t)$ where $\bar{B}_{2}=\operatorname{diag}(0,1,1)$ and $\bar{u}_{2}(t)=(1,0,0)^{\top}$.

\section{Continuous Linear Quadratic Tracker for Chua's Circuit}

Consider a controllable and observable continuoustime system represented by the following statespace system, which is applicable to each subsystem

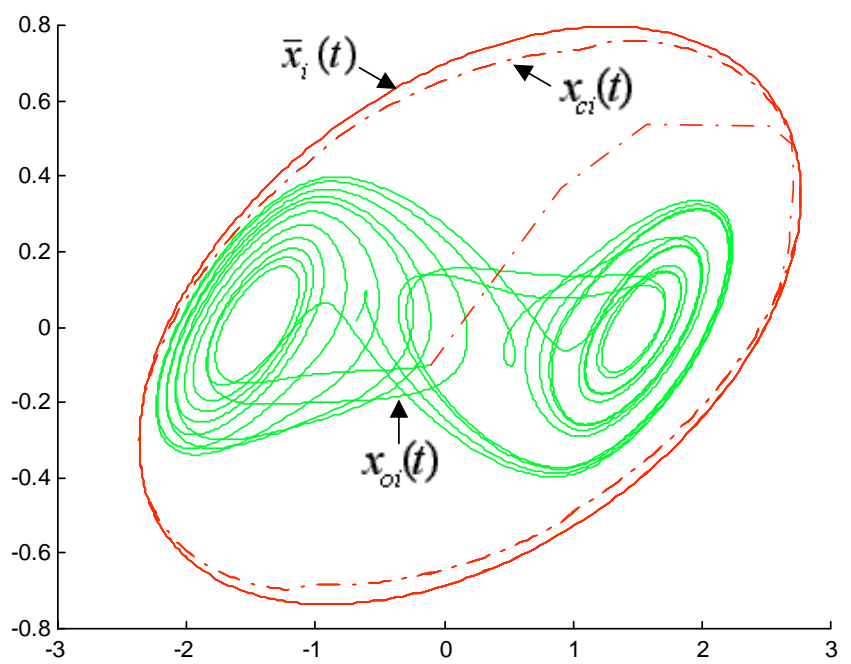

Fig. 2. $x_{o i}(t)$ : The chaotic trajectory of Chua's circuit, projected onto the $x_{1}-x_{2}$ plane; $x_{c i}(t)$ : The trajectory of Chua's circuit, projected onto the $x_{1}-x_{2}$ plane, after continuous-time feedback control is applied; $\bar{x}_{i}(t)$ : The trajectory of the approximate limit cycle, projected onto the $x_{1}-x_{2}$ plane, as the target for tracking. 


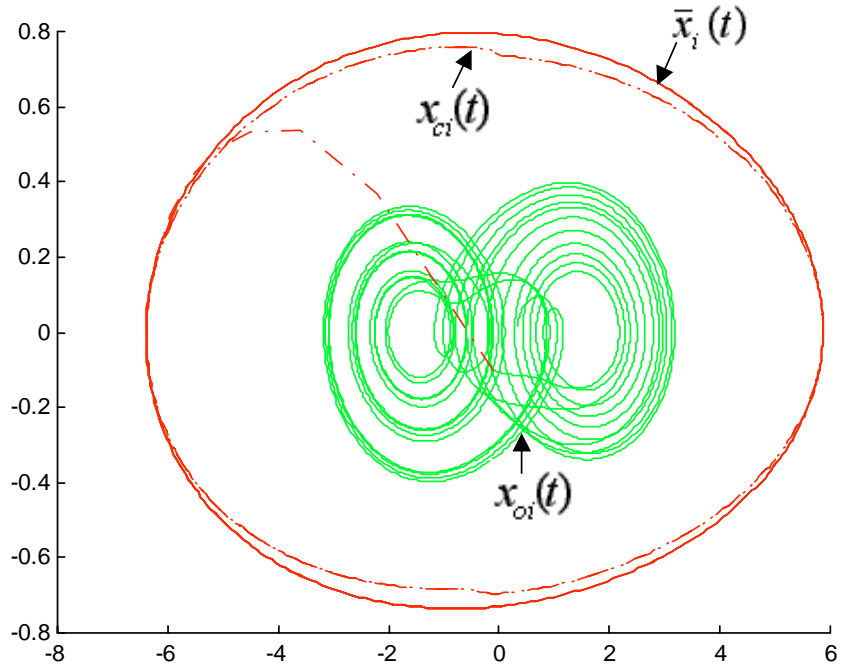

Fig. 3. $x_{o i}(t)$ : The chaotic trajectory of Chua's circuit, projected onto the $x_{3}-x_{2}$ plane; $x_{c i}(t)$ : The trajectory of Chua's circuit, projected onto the $x_{3}-x_{2}$ plane, after continuous-time feedback control is applied; $\bar{x}_{i}(t)$ : The trajectory of the approximate limit cycle, projected onto the $x_{3}-x_{2}$ plane, as the target for tracking.

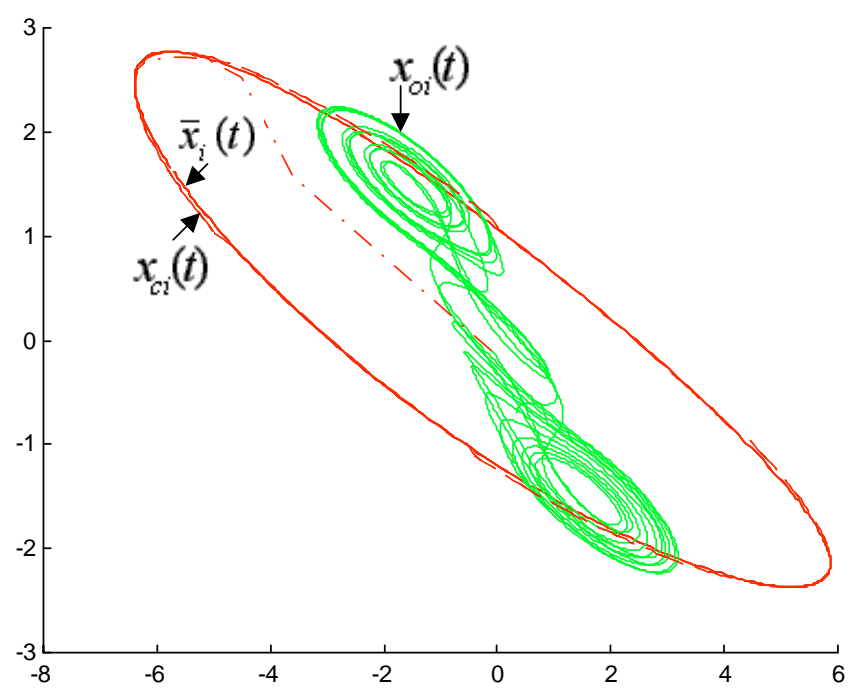

Fig. 4. $x_{o i}(t)$ : The chaotic trajectory of Chua's circuit, projected onto the $x_{3}-x_{1}$ plane; $x_{c i}(t)$ : The trajectory of Chua's circuit, projected onto the $x_{3}-x_{1}$ plane, after continuous-time feedback control is applied; $\bar{x}_{i}(t)$ : The trajectory of the approximate limit cycle, projected onto the $x_{3}-x_{1}$ plane, as the target for tracking.

(6)-(8) of the circuit:

$$
\left\{\begin{array}{l}
\dot{x}_{c}(t)=A x_{c}(t)+B u_{c}(t), \quad x_{c}(0)=x_{0}, \\
y_{c}(t)=C x_{c}(t),
\end{array}\right.
$$

where $x_{c}(t) \in R^{n}, u_{c}(t) \in R^{m}$, and $y_{c}(t) \in R^{p}$. Let $p=m$ here for the tracking purpose. For a reference input, $\Gamma(t)$, the optimal state-feedback control law [Lewis, 1986] that minimizes the performance index

$$
\begin{aligned}
J= & \int_{0}^{\infty}\left\{\left[C x_{c}(t)-\Gamma(t)\right]^{\top} Q\left[C x_{c}(t)-\Gamma(t)\right]\right. \\
& \left.+u_{c}^{\top}(t) R u_{c}(t)\right\} d t
\end{aligned}
$$

with $Q \geq 0$ and $R>0$ for system (9), is given by

$$
u_{c}(t)=-K_{c} x_{c}(t)+E_{c} \Gamma(t),
$$

where the analog feedback gain $K_{c}(t) \in R^{m \times n}$ and the forward gain $E_{c} \in R^{m \times m}$ are

$$
K_{c}=R^{-1} B^{\top} P
$$

and

$$
E_{c}=-R^{-1} B^{\top}\left[\left(A-B K_{c}\right)^{-1}\right]^{\top} C^{\top} Q,
$$

and $P$ is the solution of

$$
A^{\top} P+P A-P B R^{-1} B^{\top} P+C^{\top} Q C=0 .
$$

Note that implementation of the tracker for Chua's circuit (6)-(8) can be simplified by carrying out most of the work offline.

It has been mathematically proven via the rigorous Lyapunov stability theory [Chen \& Dong, 1993a] that the chaotic orbit of Chua's circuit can be controlled to its unstable limit cycle, where the desired feedback control can be applied to the circuit at any time. Therefore, similar mathematical analysis of the controllability of the circuit by the above optimal linear quadratic tracker is not repeated here. Instead, we only show how and how well the tracker works by showing its control of the chaotic orbit to an approximation of the unstable limit cycle of the circuit (since the exact analytic expression for the cycle does not exist). For simplicity, only the second-order approximation is used here for demonstration.

Let $\bar{\Gamma}(t)=\left(\bar{x}_{1}(t), \bar{x}_{2}(t), \bar{x}_{3}(t)\right)^{\top}$ be the unstable limit cycle of the circuit. It can be approximated by the following analytic formula [Chen \& Dong, 1993a, 1993b]

$$
\begin{aligned}
\bar{x}_{1}(t) \approx & a \cos (\xi) \cos (\omega t)-b \sin (\xi) \sin (\omega t) \\
& +c \cos (\xi) \cos (2 \omega t)-d \sin (\xi) \sin (2 \omega t) \\
\bar{x}_{2}(t) \approx & e[a \sin (\xi) \cos (\omega t)-b \cos (\xi) \sin (\omega t)] \\
& +f[c \sin (\xi) \cos (2 \omega t)-d \cos (\xi) \sin (2 \omega t)] \\
\dot{\bar{x}}_{3}(t)= & -\beta \bar{x}_{2}(t),
\end{aligned}
$$


with $a=2.6, b=1.2, c=d=0.2, e=0.6, f=0.3$, $\xi=\pi / 18, \omega=1.77, t=k T$ for $k=0,1,2, \ldots$, and $T$ is the sampling period. This second-order approximate orbit is shown also in Figs. 2-4, where $\bar{x}_{3}(0)=-6$.

Using the tracker gains (12) with $Q=2 \times 10^{3} I_{n}$ and $R=I_{m}$ in (10), the desired continuoustime state-feedback controller for tracking the target $\bar{x}(t)=\left(\bar{x}_{1}(t), \bar{x}_{2}(t), \bar{x}_{3}(t)\right)^{\top}$, which are implemented in the three continuously connected linear subsystems (6)-(8), respectively, are obtained as

$$
\begin{aligned}
& u_{1}(t)=-\left[\begin{array}{rrr}
44.0697 & 2.6119 & -0.0251 \\
0.6772 & 45.3601 & -6.4634 \\
-0.0065 & -6.4634 & 44.1055
\end{array}\right] x(t) \\
& +\left[\begin{array}{rrr}
44.7045 & 0.3037 & -0.0473 \\
-0.2653 & 41.9412 & -7.4385 \\
-0.0195 & 7.0973 & 44.0870
\end{array}\right] \bar{x}(t) \\
& x_{1}(t)>1, \\
& u_{2}(t)=-\left[\begin{array}{rrr}
45.8580 & 4.9804 & 0.3433 \\
4.9804 & 45.9862 & -6.3978 \\
0.3433 & -6.3978 & 44.1152
\end{array}\right] x(t) \\
& +\left[\begin{array}{rrr}
44.5328 & -3.7224 & 0.3190 \\
3.7620 & 41.0857 & -7.3755 \\
0.2843 & 6.9188 & 44.0956
\end{array}\right] \bar{x}(t) \\
& \left|x_{1}(t)\right| \leq 1, \\
& u_{3}(t)=-\left[\begin{array}{rrr}
-44.0697 & -2.6119 & 0.0251 \\
0.6772 & 45.3601 & -6.4634 \\
-0.0065 & -6.4634 & 44.1055
\end{array}\right] x(t) \\
& +\left[\begin{array}{rrr}
-44.7045 & -0.3037 & 0.0473 \\
-0.2653 & 41.9412 & -7.4385 \\
-0.0195 & 7.0973 & 44.0870
\end{array}\right] \bar{x}(t), \\
& x_{1}(t)<-1 .
\end{aligned}
$$

The resulting closed-loop controlled system is then given by

$$
\left\{\begin{array}{l}
\dot{x}_{c}(t)=\left[A-B K_{c}\right] x_{c}(t)+B E_{c} \bar{\Gamma}(t), \\
y_{c}(t)=C x_{c}(t)
\end{array}\right.
$$

where $C=I_{p}$. From the simulation point of view, one may simulate the time responses of a controlled system by using the following discretizations [Lewis, 1992]:

$$
\left\{\begin{aligned}
x_{c}\left(k_{f} T_{f}+T_{f}\right)= & g\left(A-B K_{c}, T_{f}\right) x_{c}\left(k_{f} T_{f}\right) \\
& +h\left(A-B K_{c}, B, T_{f}\right) E_{c} \Gamma\left(k_{f} T_{f}\right) \\
y_{c}\left(k_{f} T_{f}+T_{f}\right)= & C x_{c}\left(k_{f} T_{f}+T_{f}\right),
\end{aligned}\right.
$$

for $k_{f}=0,1,2, \ldots$, where $g(\cdot)$ and $h(\cdot)$ represent some appropriate functions of $(\cdot)$, and $\Gamma\left(k_{f} T_{f}\right)$ is to be determined to satisfy the tracking objective, $y_{c}\left(k_{f} T_{f}+T_{f}\right)=\bar{\Gamma}\left(k_{f} T_{f}+T_{f}\right)$. For simplicity of notation, let

$$
G_{c}=g\left(A-B K_{c}, T_{f}\right)=e^{\left(A-B K_{c}\right) T_{f}}
$$

and

$$
\begin{aligned}
H_{c} & =h\left(A-B K_{c}, B, T_{f}\right) \\
& =\left(e^{\left(A-B K_{c}\right) T_{f}}-I_{n}\right)\left(A-B K_{c}\right)^{-1} B,
\end{aligned}
$$

in which $\left(e^{\left(A-B K_{c}\right) T_{f}}-I_{n}\right)\left(A-B K_{c}\right)^{-1}$ as a product is always well defined, as can be easily verified by expanding the exponential term in Taylor series. The same remark applies to similar expressions below, throughout this paper.

Thus, based on the discrete final-value theorem, at the steady-state

$$
\begin{aligned}
\lim _{k_{f} \rightarrow \infty} & y_{c}\left(k_{f} T_{f}+T_{f}\right) \\
= & \lim _{z \rightarrow 1}\left(1-z^{-1}\right) Z\left\{y_{c}\left(k_{f} T_{f}+T_{f}\right)\right\},
\end{aligned}
$$

where $Z\{\cdot\}$ denotes the $Z$-transform, we have

$$
\Gamma\left(k_{f} T_{f}\right)=\bar{\Gamma}\left(k_{f} T_{f}+T_{f}\right)
$$

and

$$
E_{c}=\left[C\left(I_{n}-G_{c}\right)^{-1} H_{c}\right]^{-1} .
$$

Regarding the above discretizations, some remarks are in order. First, the gain $E_{c}$ obtained in (19) is based on the discrete final-value theorem at steady-state, but the gain $E_{c}$ shown in (12) is derived based on the optimal solution at all times. Second, when the desired gain $K_{c}$ is used and yet the desired $\Gamma\left(k_{f} T_{f}\right)$ in (18) is taken as $\Gamma\left(k_{f} T_{f}\right)=$ $\bar{\Gamma}\left(k_{f} T_{f}\right)$, then it will yield $y_{c}\left(k_{f} T_{f}+T_{f}\right)=\bar{\Gamma}\left(k_{f} T_{f}\right)$ which is a $T_{f}$-shift of the correct result. Therefore, for a large sampling period $T_{f}$, the simulated system output $y_{c}\left(k_{f} T_{f}\right)$ will be far away from the 
actual output of the given system - even if the controller gain $K_{c}$ is well designed based on the tracking objective. Notably, for a chaotic system the output responses often have rapid switchings and runnings, so that the difference between $\bar{\Gamma}\left(k_{f} T_{f}\right)$ and $\bar{\Gamma}\left(k_{f} T_{f}+T_{f}\right)$ is significantly large. As a result, the control performance is dramatically degraded, regardless of the rigorous design of the original continuous-time tracker. This is especially true for sampled-data feedback control systems, where the digital controller is held by a zero-order-hold device for a relatively long period of time. This explains why the sampling time chosen by Yang and Chua [1998] is very small ( $T=0.005 \mathrm{~s})$, where no optimality or advanced redesign technique was used. For comparison, the above digital design based on the continuous-time optimal controller showed improvement over the design method of Yang and Chua [1998]: with $T=0.015 \mathrm{~s}$, their method fails, but our simulation is successful even for $T=0.2 \mathrm{~s}$. These results are shown in Figs. 2-4. In our simulations, digital control gains (33) and (34) given below were used, with $x_{c}(0)=x_{0}=(-0.1,-0.1,-0.1)^{\top}$. A further improvement of this digital design via digital redesign will be discussed in the next section.

\section{Digital Redesign for Ordering Chaos in Chua's Circuit}

Consider a controllable and observable continuoustime system described by

$$
\left\{\begin{array}{l}
\dot{x}_{c}(t)=A x_{c}(t)+B u_{c}(t), \quad x_{c}(0)=x_{0}, \\
y_{c}(t)=C x_{c}(t),
\end{array}\right.
$$

where $x_{c}(t) \in R^{n}$ is the state, $u_{c}(t) \in R^{m}$ is the control input, $y_{c}(t) \in R^{p}$ is the output, and $(A, B, C)$ are constant matrices of appropriate dimensions. Let the controller be

$$
u_{c}(t)=-K_{c} x_{c}(t)+E_{c} \Gamma(t),
$$

where $\Gamma(t)$ is a reference input, which is assumed to be a piecewise-constant signal, and $\Gamma(t)=\Gamma(k T)$ with $T>0$ being the sampling time, $k T \leq t<$ $(k+1) T, k=0,1,2, \ldots$ The controlled system is

$$
\dot{x}_{c}(t)=A_{c} x_{c}(t)+B E_{c} \Gamma(t), \quad x_{c}(0)=x_{0},
$$

where $A_{c}=A-B K_{c}$. The corresponding discretetime model for $\Gamma(t)=\Gamma(k T)$ with $k T \leq t<(k+1) T$ in $(22)$ is

$$
x_{c}(k T+T)=G_{c} x_{c}(k T)+H_{c} E_{c} \Gamma(k T),
$$

where

$$
G_{c}=e^{A_{c} T} \quad \text { and } \quad H_{c}=\left(G_{c}-I_{n}\right) A_{c}^{-1} B .
$$

Also, let the fast sampled discrete-time model (22) for $T_{N}=T / N$, where $N$ is a positive integer, and $\Gamma(t)=\Gamma(k T)$ for $k T \leq t<(k+1) T$, be written as

$$
\begin{aligned}
x_{c}\left(k T+i T_{N}\right) & =G_{c}^{(i)} x_{c}(k T)+H_{c}^{(i)} \Gamma(k T), \\
i & =1,2, \ldots, N,
\end{aligned}
$$

where

$$
G_{c}^{(i)}=\left(e^{A_{c} T_{N}}\right)^{i} \quad \text { and } \quad H_{c}^{(i)}=\left(G_{c}^{(i)}-I_{n}\right) A_{c}^{-1} B .
$$

Moreover, let the state equation of the continuoustime system (20) with a piecewise-constant input, $u_{d}(t)$, be represented by

$$
\left\{\begin{array}{l}
\dot{x}_{d}(t)=A x_{d}(t)+B u_{d}(t), \quad x_{d}(0)=x_{0}, \\
y_{d}(t)=C x_{d}(t)
\end{array}\right.
$$

where $u_{d}(t)=u_{d}(k T)$ for $k T \leq t<(k+1) T$. Then, let the digital controller for system (25), with $\Gamma(t)=\Gamma(k T)$ for $k T \leq t<(k+1) T$, be

$$
u_{d}(t)=-K_{d} x_{d}(k T)+E_{d} \Gamma(k T),
$$

where $K_{d} \in R^{m \times n}$ and $E_{d} \in R^{m \times m}$ are the feedback and feedforward digital gains, respectively.

Thus, the designed closed-loop sampled-data system (25) and (26) is

$\dot{x}_{d}(t)=A x_{d}(t)-B K_{d} x_{d}(k T)+B E_{d} \Gamma(k T)$.

A zero-order-hold device is used for (27). The corresponding discrete-time model of the sampleddata feedback control system (27) is

$$
x_{d}(k T+T)=\left(G-H K_{d}\right) x_{d}(k T)+H E_{d} \Gamma(k T),
$$

where

$$
G=e^{A T} \quad \text { and } \quad H=\left(G-I_{n}\right) A^{-1} B .
$$

The process of finding digital controller gains $\left(K_{d}, H_{d}\right)$ in $(26)$ from the analog gains $\left(K_{c}, E_{c}\right)$ in $(21)$, so that the closed-loop state $x_{d}(t)$ in (27) can closely match the closed-loop state in (22), is called the state-matching digital redesign. 
To match the closed-loop state $x_{d}(t)$ in (27) to the closed-loop state in (22) at each sampling instant, $t=k T$, it is required that

$$
G_{c}=G-H K_{d} \quad \text { and } \quad H_{c} E_{c}=H E_{d},
$$

where the $m \times n$ feedback gain $K_{d}$ and the $m \times m$ feedforward gain $E_{d}$ are unknown matrices to be determined. To explore the intersampling behavior of the original analogously designed system (22) and the digitally redesigned system (27), we first briefly review the development of the Chebyshev quadrature method [Shieh et al., 1998] as follows.

The discrete-time model of the sampled-data system (22) for $\Gamma(t)=\Gamma(k T)$, with $k T \leq t<$ $(k+1) T$, can be written as

$$
\begin{aligned}
x_{c}(k T+T)= & G x_{c}(k T) \\
& -\int_{k T}^{k T+T} e^{A(k T+T-\lambda)} B K_{c} x_{c}(\lambda) d \lambda \\
& +H E_{c} \Gamma(k T) .
\end{aligned}
$$

To reduce the error in the convolution integral over each time interval $T$, Shieh et al. [1998] used a Chebyshev quadrature formula to evaluate the integral term in (30). The general Chebyshev quadrature formula has the form

$$
\int_{a}^{b} w(\lambda) f(\lambda) d \lambda \approx W \sum_{i=0}^{N} f\left(\lambda_{i}\right),
$$

where $w(\lambda)$ is a constant weighting function defined on $[a, b]$, and $W$ is the weighting factor

$$
W=\frac{1}{N+1} \int_{a}^{b} w(\lambda) d \lambda
$$

with $f\left(\lambda_{i}\right)$ being the values of $f(\cdot)$ at the partition points $\lambda_{i}=a+i(b-a) / N, i=0,1, \ldots, N$.
The above formulation can be extended to the matrix convolution integral shown in (30) as follows:

$$
\begin{aligned}
\int_{k T}^{k T+T} & e^{A(k T+T-\lambda)} B K_{c} x_{c}(\lambda) d \lambda \\
& =\frac{1}{N+1} H K_{c} \sum_{i=0}^{N} x_{c}\left(k T+i T_{N}\right)
\end{aligned}
$$

where the intersampling state, $x_{c}\left(k T+i T_{N}\right)$, for the piecewise-constant reference input, $\Gamma(t)=\Gamma(k T)$, is shown in (24). Substituting this equation into (30) yields

$$
\begin{aligned}
x_{c}(k T+T) & \\
= & G x_{c}(k T)-H\left(\frac{1}{N+1} K_{c} \sum_{i=0}^{N} G_{c}^{(i)}\right) x_{c}(k T) \\
& +H\left(I_{m}-\frac{1}{N+1} K_{c} \sum_{i=0}^{N} H_{c}^{(i)}\right) E_{c} \Gamma(k T) .
\end{aligned}
$$

Now, let the closed-loop states $x_{c}(k T+T)$ and $x_{c}(k T)$ in (31), which involve the intersampling states $x_{c}\left(k T+i T_{N}\right)$ for $i=0,1, \ldots, N$, be equal to the desired closed-loop state $x_{d}(k T+T)$ and $x_{d}(k T)$ in (28), respectively. Then we have

$$
\begin{aligned}
G-H K_{d} & =G-H\left(\frac{1}{N+1} K_{c} \sum_{i=0}^{N} G_{c}^{(i)}\right) \\
H E_{d} & =H\left(I_{m}-\frac{1}{N+1} K_{c} \sum_{i=0}^{N} H_{c}^{(i)}\right) E_{c} .
\end{aligned}
$$

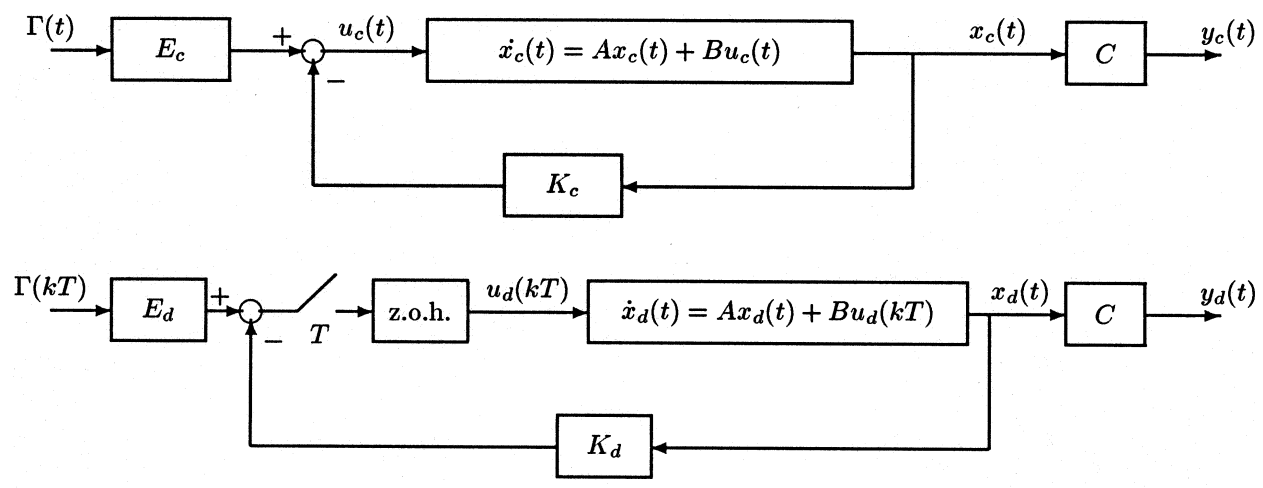

Fig. 5. (a) Continuous-time control system; (b) Digital control system. 
Solving Eq. (32) yields the desired digital control gains, as

$$
\begin{aligned}
K_{d} & =\frac{1}{N+1} K_{c} \sum_{i=0}^{N} G_{c}^{(i)}=\frac{1}{N+1} K_{c}\left(\left[G_{c}^{(1)}-I_{n}\right]^{-1}\left(G_{c}-I_{n}\right)+G_{c}\right), \\
E_{d} & =\left(I_{m}-\frac{1}{N+1} K_{c} \sum_{i=0}^{N} H_{c}^{(i)}\right) E_{c} .
\end{aligned}
$$

To this end, letting $N \rightarrow \infty$ leads to

$$
\begin{aligned}
K_{d} & =\lim _{N \rightarrow \infty} \frac{1}{N+1} K_{c}\left(\left[G_{c}^{(i)}-I_{n}\right]^{-1}\left(G_{c}-I_{n}\right)+G_{c}\right) \\
& =K_{c} \lim _{N \rightarrow \infty}\left[\frac{N+1}{N}\left(A_{c} T+\frac{1}{2 !} \frac{\left(A_{c} T\right)^{2}}{N}+\cdots\right)\right]^{-1}\left(G_{c}-I_{n}\right) \\
& =K_{c}\left(A_{c} T\right)^{-1}\left(G_{c}-I_{n}\right) .
\end{aligned}
$$

and

$$
\begin{aligned}
E_{d} & =E_{c}-\lim _{N \rightarrow \infty} \frac{1}{N+1} K_{c}\left(\sum_{i=0}^{N} G_{c}^{(i)}-(N+1) I_{n}\right) A_{c}^{-1} B E_{c} \\
& =E_{c}+\left(K_{c}-\lim _{N \rightarrow \infty} \frac{1}{N+1} K_{c} \sum_{i=0}^{N} G_{c}^{(i)}\right) A_{c}^{-1} B E_{c} \\
& =E_{c}+\left(K_{c}-K_{d}\right) A_{c}^{-1} B E_{c} .
\end{aligned}
$$

It should be remarked that the integral in (30) uses $N$ intersampling states of the closedloop system (22) and the accuracy of the integration depends on the number of intersampling states involved. Since infinitely many sampling states $(N \rightarrow \infty)$ are used to determine the digital control gains in (28), it can be expected that the digitally redesigned sampled-data state $x_{d}(t)$ in (27) is able to capture the intersampled behavior of the original continuous-time controlled state $x_{c}(t)$ in $(22)$, for the piecewise-constant reference input $\Gamma(t)=\Gamma(k T)$, for all $k=0,1,2, \ldots$

Consider the special case of $\operatorname{rank}(B)=n$, which is the dimension of the state $x_{c}(t)$. An explicit formula, named the full-rank formula, for state matching $x_{c}(k T)=x_{d}(k T), k=0,1,2, \ldots$, is given by

$$
K_{d}=H^{-1}\left(G-G_{c}\right) \text { and } E_{d}=H^{-1}\left(H_{c} E_{c}\right) .
$$

Based on the observation described above, the $K_{d}$ in (32) and the $E_{d}$ in (33) used in this paper work also for the general case where $\operatorname{rank}(B)$ may not equal to $n$. It captures the intersampled behavior of the original continuous-time controlled state $x_{c}(t)$ shown in (21). However, the tracker obtained based on the full-rank formula does not have these two advantages in general.

Before leaving this section to the next section on simulation studies and comparison, another pair of digitally redesigned state-feedback and feedforward gain matrices $\left(\tilde{K}_{d}, \tilde{E}_{d}\right)$, derived in [Shieh et al., 1992], are listed here:

$$
K_{d}=\left(I_{m}+K_{c} H^{(\nu)}\right)^{-1} K_{c} G^{(\nu)}
$$

and

$$
E_{d}=\left(I_{m}+K_{c} H^{(\nu)}\right)^{-1} E_{c}
$$

where

$$
G^{(\nu)}=e^{A \nu T}
$$

and $\quad H^{(\nu)}=\int_{0}^{T} e^{A \nu \tau} B d \tau=\left(G^{(\nu)}-I_{n}\right) A^{-1} B$.

In these formulas, the choice of the tuning parameter $\nu$ in (35) depends on the specified sampling interval $T$ and the desired closeness of the trajectory $x_{d}(t)$ of the redesigned digital system (27) to $x_{c}(t)$, the orbit of the original continuous-time system (22). The following performance index is suggested as a design criterion for the selection of this 
turning parameter, $\nu$ :

$$
J(\nu)=\sum_{i=1}^{n} \int_{0}^{t_{f}}\left|x_{c i}(t)-x_{d i}(t)\right| d t,
$$

where $t_{f}$ is the desired finite terminal time for the minimization.

\section{Computer Simulation and Comparison Examples}

For comparison purpose, we have chosen a long sampling period, $T=0.015 \mathrm{~s}$, for which the sampled-data control method of Yang and Chua [1998], which was discussed in Sec. 3, fails.

With a fairly long sampling time, $T=0.2 \mathrm{~s}$, the digitally controlled Chua's circuit is given by the following three continuously connected subsystems:

$$
\begin{gathered}
\dot{x}_{d}(t)=\left[\begin{array}{ccc}
-\alpha\left(1+m_{0}\right) & \alpha & 0 \\
1 & -1 & 1 \\
0 & -\beta & 0
\end{array}\right] x_{d}(t) \\
+\left[\begin{array}{ccc}
-\alpha\left(m_{1}-m_{0}\right) & 0 & 0 \\
0 & 1 & 0 \\
0 & 0 & 1
\end{array}\right] u_{d}(k T), \\
x_{1}(t)>1,
\end{gathered}
$$

where

$$
\begin{aligned}
u_{d}(k T)= & -\left[\begin{array}{rrr}
1.2774 & 0.2451 & 0.0414 \\
0.0287 & 4.8760 & 0.0919 \\
-0.0028 & -1.4973 & 4.7466
\end{array}\right] x_{d}(k T) \\
& +\left[\begin{array}{rrr}
1.9403 & -1.8871 & 0.0395 \\
-0.9633 & 5.3148 & -0.9046 \\
0.0096 & 11.5726 & 4.7500
\end{array}\right] \\
& \times \bar{\Gamma}(k T+T) \\
\dot{x}_{d}(t)= & {\left[\begin{array}{rrr}
-\alpha\left(1+m_{1}\right) & \alpha & 0 \\
1 & -1 & 1 \\
0 & -\beta & 0
\end{array}\right] x(t) } \\
& +\left[\begin{array}{lll}
1 & 0 & 0 \\
0 & 1 & 0 \\
0 & 0 & 1
\end{array}\right] u_{d}(k T), \quad\left|x_{1}(t)\right| \leq 1, \quad(37)
\end{aligned}
$$

where

$$
\begin{aligned}
u_{d}(k T)= & -\left[\begin{array}{rrr}
5.0589 & 0.9363 & 0.1562 \\
0.1217 & 4.8884 & 0.0932 \\
0.1326 & -1.4669 & 4.7529
\end{array}\right] \\
& \times x_{d}(k T) \\
& +\left[\begin{array}{rrr}
3.8055 & -7.0721 & 0.1507 \\
-0.9039 & 5.1533 & -0.9033 \\
0.0708 & 11.2164 & 4.7561
\end{array}\right] \\
& \times \bar{\Gamma}(k T+T)
\end{aligned}
$$$$
\dot{x}_{d}(t)=\left[\begin{array}{ccc}
-\alpha\left(1+m_{0}\right) & \alpha & 0 \\
1 & -1 & 1 \\
0 & -\beta & 0
\end{array}\right] x(t)
$$$$
+\left[\begin{array}{ccc}
\alpha\left(m_{1}-m_{0}\right) & 0 & 0 \\
0 & 1 & 0 \\
0 & 0 & 1
\end{array}\right] u_{d}(k T) \text {, }
$$$$
x_{1}(t)<-1 \text {, }
$$

where

$$
\begin{aligned}
u_{d}(k T)= & -\left[\begin{array}{rrr}
-1.2774 & -0.2451 & -0.0414 \\
0.0287 & 4.8760 & 0.0919 \\
-0.0028 & -1.4973 & 4.7466
\end{array}\right] \\
& \times x_{d}(k T) \\
& +\left[\begin{array}{rrr}
-1.9403 & 1.8871 & -0.0395 \\
-0.9633 & 5.3148 & -0.9046 \\
0.0096 & 11.5726 & 4.7500
\end{array}\right] \\
& \times \bar{\Gamma}(k T+T) .
\end{aligned}
$$

The initial condition used is $x_{d}(0)=(-0.1$, $-0.1,-0.1)^{\top}$, and the reference is the second-order approximation of the unstable limit cycle given in Sec. 3 above. The control performance under this digitally redesigned controller is visualized by Figs. 6-8, while Figs. 9-11 are the control input signals.

For comparison, the simulation results obtained based on the parameter tuning approach (35), with $\nu=0.9$ determined, has also been verified, which are about the same as those obtained in Figs. 6-8. The main difference is that the tuning method relies on a trial-and-error procedure to find 


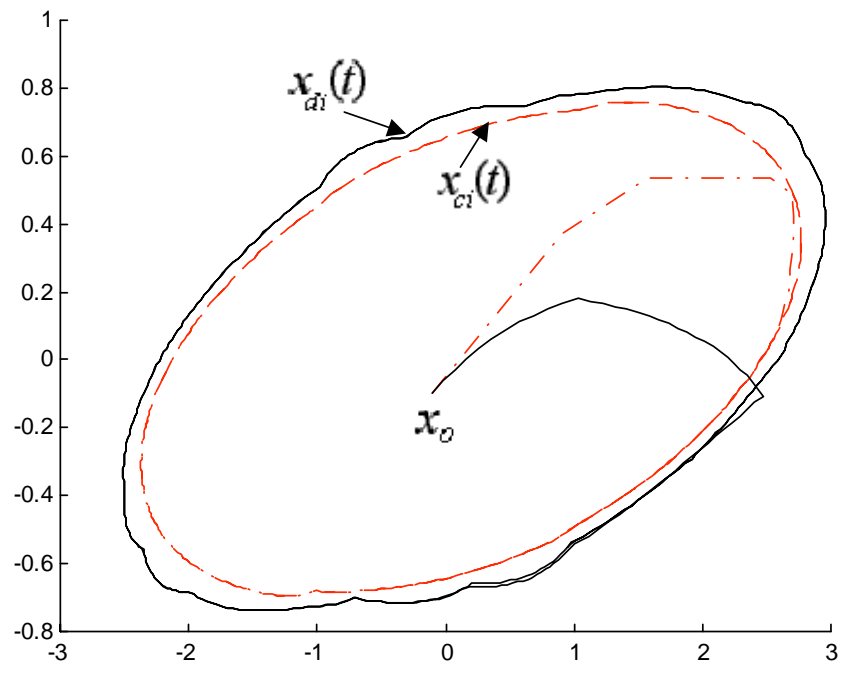

Fig. 6. $x_{c i}(t)$ : The trajectory of Chua's circuit, projected onto the $x_{1}-x_{2}$ plane, after continuous-time feedback control is applied; $x_{d i}(t)$ : The trajectory of Chua's circuit, projected onto the $x_{1}-x_{2}$ plane, after digitally redesigned control is applied, with $T=0.2 \mathrm{~s}$.

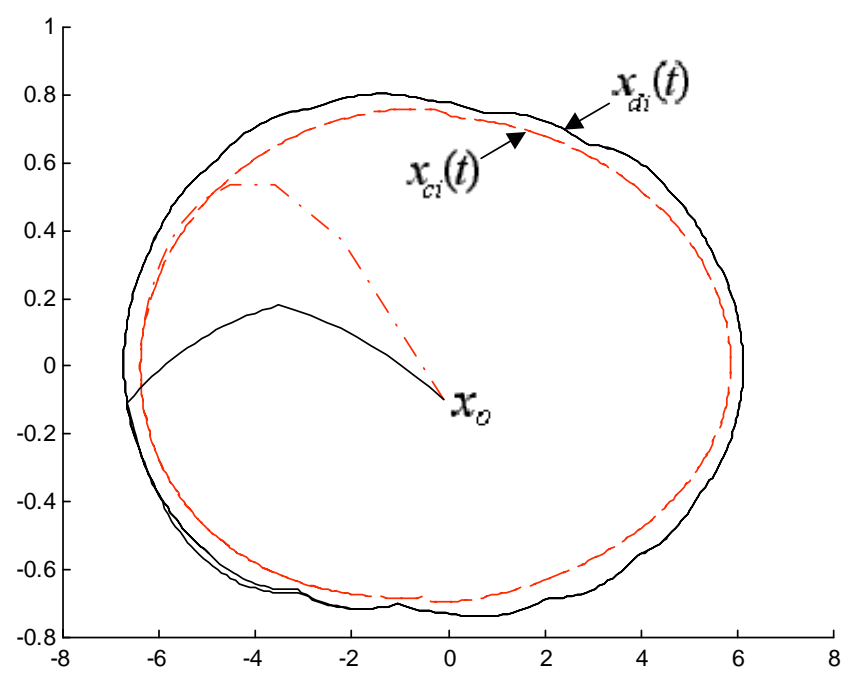

Fig. 7. $x_{c i}(t)$ : The trajectory of Chua's circuit, projected onto the $x_{3}-x_{2}$ plane, after continuous-time feedback control is applied; $x_{d i}(t)$ : The trajectory of Chua's circuit, projected onto the $x_{3}-x_{2}$ plane, after digitally redesigned control is applied, with $T=0.2 \mathrm{~s}$.

a good parameter $\nu$, while the redesign method suggested and used in this paper provides a systematic approach.

All the simulation results have shown that the digitally redesigned sampled-data feedback control laws are effective in guiding the chaotic trajectories of the chaotic Chua's circuit to the intended (approximation of) unstable limit cycle, even when the sampling period is very long $(T=0.2 \mathrm{~s}$ in these simulations).

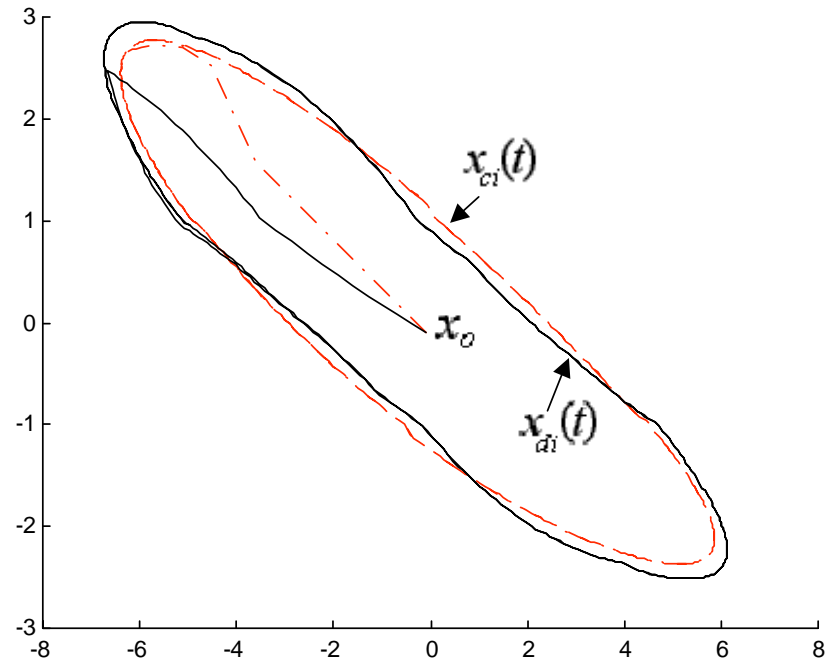

Fig. 8. $x_{c i}(t)$ : The trajectory of Chua's circuit, projected onto the $x_{3}-x_{1}$ plane, after continuous-time feedback control is applied; $x_{d i}(t)$ : The trajectory of Chua's circuit, projected onto the $x_{3}-x_{1}$ plane, after digitally redesigned control is applied, with $T=0.2 \mathrm{~s}$.

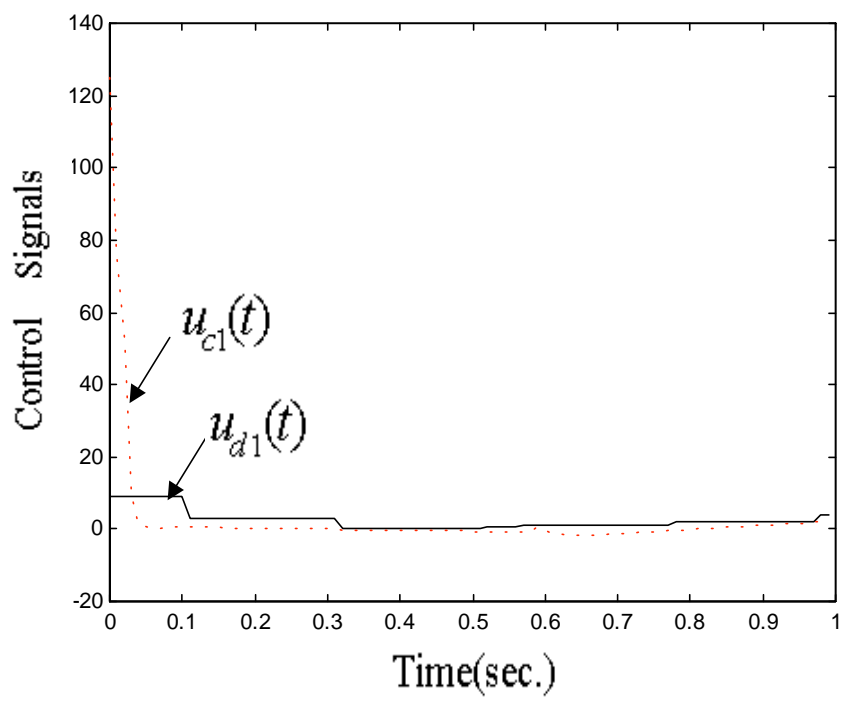

Fig. 9. $u_{c 1}(t)$ : Control signal in continuous-time feedback control; $u_{d 1}(t)$ : Control signal in digitally redesigned feedback control; $u_{d 1}(0) \approx 1 / 12 u_{c 1}(0)$ which overcomes the saturation problem.

\section{Conclusions}

An approach to ordering the chaotic Chua's circuit via digital redesign of an existing analog controller, using a relatively long sampling period, has been suggested and simulated in this paper with comparisons. It has been shown that when the sampling period is relatively long, some existing sampled-data feedback control method fails, but the digitally redesigned method used in this paper 


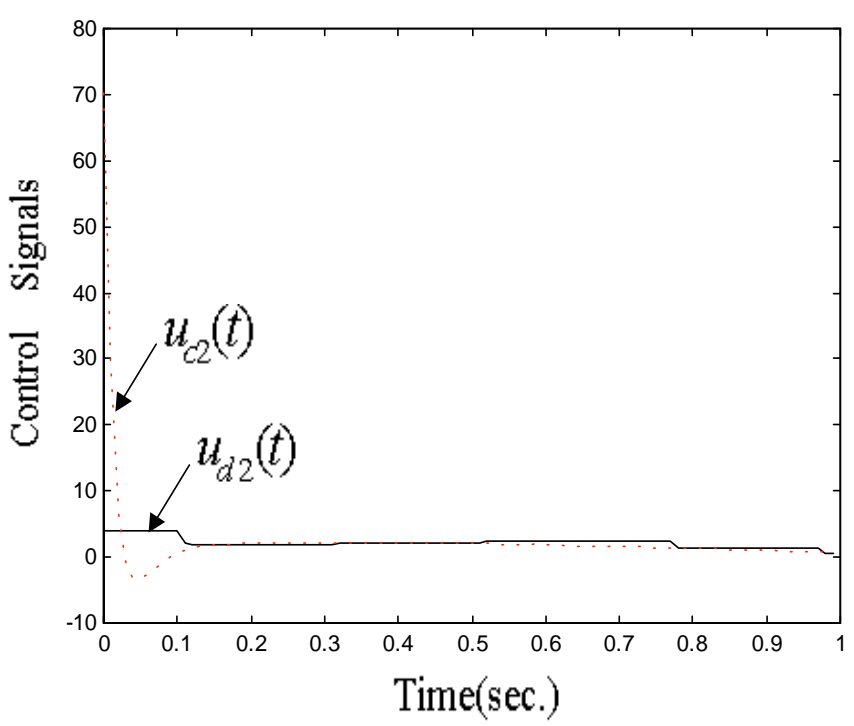

Fig. 10. $u_{c 2}(t)$ : Control signal in continuous-time feedback control; $u_{d 2}(t)$ : Control signal in digitally redesigned feedback control; $u_{d 2}(0) \approx 1 / 12 u_{c 2}(0)$ which overcomes the saturation problem.

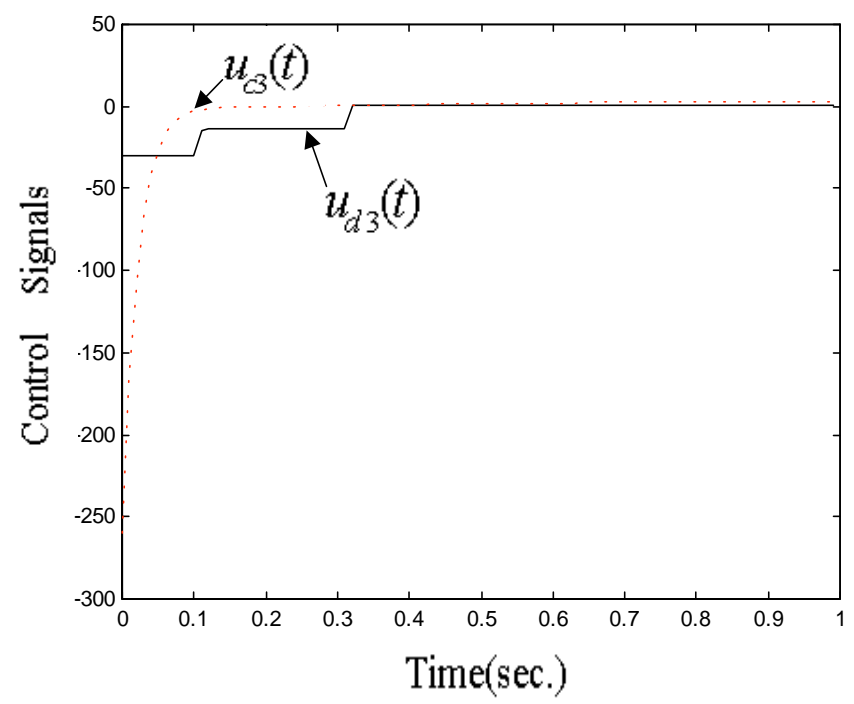

Fig. 11. $u_{c 3}(t)$ : Control signal in continuous-time feedback control; $u_{d 3}(t)$ : Control signal in digitally redesigned feedback control; $u_{d 3}(0) \approx 1 / 9 u_{c 3}(0)$ which overcomes the saturation problem.

succeeds very well. The main reason is that during a long sampling period, a chaotic orbit usually has some abrupt qualitative changes, so the controller has to be effective enough to handle such significant variations or sensitivity of chaos. Our digital redesign method has proven successful for Chua's chaotic circuit in this paper. It is our hope that this digital redesign method can also be applied to other continuous-time chaotic dynamical systems.

\section{References}

Chen, G. \& Dong, X. [1993a] "Controlling Chua's circuit," J. Circuits Syst. Comput. 3, 139-149.

Chen, G. \& Dong, X. [1993b] "On feedback control of chaotic continuous-time systems," IEEE Trans. Circuits Syst. (I) 40, 591-601.

Chen, G. \& Dong, X. [1998] From Chaos to Order: Methodologies, Perspectives and Applications (World Scientific, Singapore)

Chen, G. (ed.) [1999] Controlling Chaos and Bifurcations in Engineering Systems (CRC Press, Boca Raton).

Chen, G. \& Ogorzalek, M. J. [2000] "Theme issue on control and synchronization of chaos," Int. J. Bifurcation and Chaos 10(3\&4).

Dedieu, H. \& Ogorzalek, M. J. [1994] "Controlling chaos in Chua's circuit via sampled inputs," Int. J. Bifurcation and Chaos 4, 447-455.

Kuo, B. C. [1980] Digital Control Systems (Holt, Rinehart and Winston, NY).

Lewis, F. L. [1986] Optimal Control (John Wiley, NY).

Lewis, F. L. [1992] Applied Optimal Control and Estimation: Digital Design and Implementation (PrenticeHall, NJ).

Shieh, L. S., Zhao, X. M. \& Zhang, J. L. [1989] "Locally optimal-digital redesign of continuous-time systems," IEEE Trans. Indust. Electron. 36, 511-515.

Shieh, L. S., Zhang, J. L. \& Coleman, N. P. [1991] "Optimal digital redesign of continuous-time controllers," Comput. Math. Appl. 22, 25-35.

Shieh, L. S., Chen, G. \& Tsai, J. S. H. [1992a] "Hybrid suboptimal control of multi-rate multi-loop sampleddata systems," Int. J. Syst. Sci. 23, 839-854.

Shieh, L. S., Zhang, J. L. \& Sunkel, J. W. [1992b] "A new approach to the digital redesign of continuoustime controllers," Contr. - Th. Adv. Techn. 8, 37-57.

Shieh, L. S., Wang, W. M. \& Appu Panicker, M. K. [1998] "Design of PAM and PWM digital controllers for cascaded analog systems," ISA - Trans. 37, 201-213.

Tsai, J. S. H., Shieh, L. S., Zhang, J. L. \& Coleman, N. P. [1989] "Digital redesign of pseudo-continuoustime suboptimal regulators for large-scale discrete system," Contr. - Th. Adv. Techn. 5, 37-65.

Tsai, J. S. H., Chen, C. M. \& Shieh, L. S. [1991] "Digital redesign of the cascaded continuous-time controller: Time-domain approach," Contr. - Th. Adv. Techn. 17, 643-661.

Xu, J., Shieh, L. S. \& Chen, G. [1996] "Digital redesign of the chaotic Chua's circuit," IEEE Trans. Aerosp. Electron. Syst. 32, 1488-1499.

Yang, T. \& Chua, L. O. [1998] "Control of chaos using sampled-data feedback control," Int. J. Bifurcation and Chaos 8, 2433-2438. 